\title{
Mathematical modeling of space-time variations in acoustic transmission and scattering from schools of swim bladder fish (FY14 Annual Report)
}

\author{
Christopher Feuillade \\ Pontificia Universidad Católica de Chile \\ Facultad de Física \\ Av. Vicuña Mackenna 4860 \\ Santiago, Chile \\ phone: +562354 4800 fax: +5623544491 email: chris.feuillade@ gmail.com
}

Award Number: N00014-111-0161

\section{LONG-TERM GOALS}

The goal is the development of a time-domain acoustical method for investigating the spatial and temporal stochastic variations in fish density within fish schools, and thereby to study the statistical fluctuations in the scattering of sound from these objects.

\section{OBJECTIVES}

The objective of this research is to develop a complete time-domain theory of acoustic scattering from, and propagation through, schools of swim bladder fish at and near the swim bladder resonance frequency, including multiple scattering and coherent interaction effects between the individual fish. This should then lead to a prescriptive capability for modeling the evolution of sound pulses as they are scattered from, and pass through, fish schools. Subsequently, it should be possible to identify discrete segments of the signal with different sections of the fish school, and to develop an enhanced

understanding of signal scattering, and extinction, by the school, and the fluctuations in these properties.

\section{APPROACH}

The personnel participating in this work at the present time are: Principal Investigator: Christopher Feuillade - Ph. D. (Physics), Manchester, UK, 1977. (Visiting Professor, Pontificia Universidad Católica de Chile); Assistant: Maria Paz Raveau Morales - Civil Engineer in Sound and Acoustics INACAP, Chile, 2009. (Doctoral student, Pontificia Universidad Católica de Chile), Simon Alfaro Jimenez (Undergraduate student, INACAP), Jorge Antonio Cellio (Undergraduate student, INACAP).

(1) Steady-state solution for a school: In 1996, a low-frequency scattering model for fish schools was introduced (see Ref. 1) using a formalism based upon the harmonic solution of sets of coupled differential equations. It incorporates a verified swim bladder scattering kernel for the individual fish, includes multiple scattering interactions between the fish, and calculates the aggregate scattering field by coherent summation. In this work, the Love swim bladder model is used as the kernel (Ref. 2). 


\section{Report Documentation Page}

Form Approved

OMB No. 0704-0188

Public reporting burden for the collection of information is estimated to average 1 hour per response, including the time for reviewing instructions, searching existing data sources, gathering and maintaining the data needed, and completing and reviewing the collection of information. Send comments regarding this burden estimate or any other aspect of this collection of information,

including suggestions for reducing this burden, to Washington Headquarters Services, Directorate for Information Operations and Reports, 1215 Jefferson Davis Highway, Suite 1204, Arlington

VA 22202-4302. Respondents should be aware that notwithstanding any other provision of law, no person shall be subject to a penalty for failing to comply with a collection of information if it

does not display a currently valid OMB control number.

1. REPORT DATE

30 SEP 2014

4. TITLE AND SUBTITLE

Mathematical Modeling of Space-time Variations in Acoustic Transmission and Scattering from Schools of Swim Bladder Fish (FY14 Annual Report)

6. $\operatorname{AUTHOR}(\mathrm{S})$

7. PERFORMING ORGANIZATION NAME(S) AND ADDRESS(ES)

Pontifical Catholic University of Chile ,Av. Vicuna Mackenna 4860,Santiago, Chile,

9. SPONSORING/MONITORING AGENCY NAME(S) AND ADDRESS(ES)
3. DATES COVERED

00-00-2014 to 00-00-2014

5a. CONTRACT NUMBER

5b. GRANT NUMBER

5c. PROGRAM ELEMENT NUMBER

5d. PROJECT NUMBER

5e. TASK NUMBER

5f. WORK UNIT NUMBER

8. PERFORMING ORGANIZATION

REPORT NUMBER

10. SPONSOR/MONITOR'S ACRONYM(S)

11. SPONSOR/MONITOR'S REPORT

NUMBER(S)

12. DISTRIBUTION/AVAILABILITY STATEMENT

Approved for public release; distribution unlimited

13. SUPPLEMENTARY NOTES

14. ABSTRACT

15. SUBJECT TERMS

16. SECURITY CLASSIFICATION OF:

a. REPORT

b. ABSTRACT

unclassified

unclassified

c. THIS PAGE

unclassified
17. LIMITATION OF ABSTRACT

Same as

Report (SAR)
18. NUMBER OF PAGES

7 19a. NAME OF

RESPONSIBLE PERSON 
Solutions are obtained by solving a matrix equation $\mathbf{M v}=\mathbf{p}$, where $\mathbf{v}=\left\{\bar{v}_{1}, \ldots, \bar{v}_{n}, \ldots, \bar{v}_{N}\right\}$ and $\mathbf{p}=\left\{-P_{1} e^{i \phi_{1}}, \ldots,-P_{n} e^{i \phi_{n}}, \ldots,-P_{N} e^{i \phi_{N}}\right\}$ are column vectors containing the steady-state volume oscillation amplitudes for the individual bladders, and the external fields applied to them (where $P_{n}$ and $\phi_{n}$ are the amplitude and phase of the external field incident on the $n$-th fish swim bladder), respectively. If there are $N$ fish in the school, $\mathbf{M}$ is an $N \times N$ matrix with elements:

$$
M_{n n}=\kappa_{n}-\omega^{2} m_{n}+i \omega b_{n} \quad ; \quad M_{n j}=\frac{-\omega^{2} \rho e^{-i k s_{j n}}}{4 \pi s_{j n}} \quad(n \neq j) .
$$

Each diagonal term [i.e., $M_{j j}(j=1, \ldots, N)$ ] describes the resonance behavior of an individual swim bladder. The quantities $m_{n}, b_{n}, \kappa_{n}$, etc., are varied to allow for different values for the swim bladder radii, damping, etc., in order to represent a diverse range of individual fish properties within the school. Every off-diagonal element [i.e., $\left.M_{n j}(j, n=1, \ldots, N), n \neq j\right)$ ] describes the radiative coupling between two of the bladders, where $s_{j n}$ denotes the separation distance between the centers of the $j$-th and $n$-th bladders, etc.

The solution of the matrix equation (i.e., $\mathbf{v}=\mathbf{M}^{-1} \mathbf{p}$ ) enables the description of steady-state scattering from the whole ensemble of swim bladders as a function of the external field amplitude and frequency. Once the solutions $\bar{v}_{n}$ are found for the individual swim bladders, the total scattered pressure field for the whole school may be readily obtained, for any azimuthal angle, using coherent summation, i.e.,

$$
p_{s}=-\frac{\rho \omega^{2}}{4 \pi} \sum_{n=1}^{N} \frac{\bar{v}_{n} e^{-i k r_{n}}}{r_{n}} \approx \frac{P_{0}}{r} \mathbf{f}_{s} \Longrightarrow \mathbf{f}_{s}(k, \theta, \phi)=\frac{-\rho \omega^{2}\left[\sum_{n=1}^{N} \bar{v}_{n} e^{-i k r_{n}}\right]}{4 \pi P_{0}},
$$

where $r_{n}$ is the distance between the $n$-th swim bladder and a point receiver, located in the far-field. For a school containing multiple fish, the ensemble scattered field in any direction will be affected by coherent interactions between the scattered fields from the individual fish. For this reason, the phase factor $e^{-i k r_{n}}$ for each swim bladder must be included inside the summation of Eq. (2). The right hand equation of Eq. (2) defines a scattering amplitude $\mathbf{f}_{s}$ for the whole school ensemble. The scattered pressure field can be obtained for a receiver placed at any arbitrary orientation with respect to the fish school, and for any bistatic angle with respect to the acoustic source, including both back and forward scattering geometries.

The steady-state volume oscillation amplitudes $\bar{v}_{n}$ are initially defined, in the original coupled differential equations, to include all radiative interaction (i.e., multiple scattering) processes between the swim bladders. The use of the $\bar{v}_{n}$ (obtained via $\mathbf{v}=\mathbf{M}^{-1} \mathbf{p}$ ) to calculate the total scattered pressure of the fish school, by Eq. (2), therefore inherently incorporates modifications of the scattered pressure as a result of multiple scattering.

(2) Time-domain solution for a school: The aim of this research is to obtain explicit analytic time-domain solutions of a more generalized form of the coupled differential equations for arbitrary time-dependent external input fields, i.e., $\left[P_{1}(t), \cdots, P_{n}(t), \cdots, P_{N}(t)\right]$, which are not necessarily harmonic. Two main approaches are currently being investigated for solving the time-domain equations: (a) reformulating the $N$ second-order differential equations for $N$ fish as an equivalent set of $2 N$ first-order differential equations, which are then treated as state equations which can be analytically solved using eigen-decomposition techniques; (b) using numerical techniques to march out a solution of the coupled equations computationally.

(3) Comparison of predictions made using both the time-independent solutions, and the time-domain implementation, against available experimental data, and also against simulated fish school scattering 
data produced by a biological model of fish schooling behavior. The purpose is to then to use these solutions to describe the evolution of sound pulses as they are scattered from, and pass through, fish schools, and to identify discrete segments of the acoustic signal with different sections of the fish school, leading to an improved understanding of signal scattering, signal extinction, and their fluctuations.

\section{WORK COMPLETED}

(1) During FY14 we continued an analysis of the comparative results obtained by investigating the forward scattered field of a fish school, rather than the back scattered field, and thereby the sound extinction due to the school, using both the current steady-state model (Ref. 1), and an effective medium approach, to study the acoustic field. In forward scattering, both methods indicate similar results, predicting significantly larger estimates of fish numbers than corresponding back scattering studies. In back scattering, we have shown that the effective medium method fails to give an accurate description of scattering from schools at a particular frequency, which is dependent on the depth of the school, the size of the swim bladder, and the spacing between fish. This failure has not been seen in forward scattering.

(2) We have developed a time domain fish school scattering model which can be used to describe stochastic variations in the scattered signals from schools. The solution is obtained by solving the coupled equations of Ref 1., using a robust numerical method to march the solution forward in time. The intention is to use this new model to analyze experimental data from other participants in the ONR BRC, Fish Acoustics program, and also simulated data, using a new model of fish schooling behavior (see 3 below).

(3) We have developed a model of fish schooling behavior, based on up to date biological modeling of collective fish movements. This has already been coupled to our current steady-state school scattering model (Ref. 1), and in the coming year will be coupled to the new time-domain scattering model.

\section{RESULTS}

(a) Comparison of the back scattered and forward scattered field with data

The following figures show the results of comparing predictions obtained via Eq. (2) with data obtained in both back scattering, and forward scattering, geometries (Ref. 3). Figure 1(a) shows a comparison with back scattering data, obtained by Holliday (Ref. 4) from schools of anchovy. The input parameters to the scattering model for the anchovies, i.e., the fish size, depth, spacing, school size and shape, etc., were taken from Holliday's article. The vertical axis shows the target strength of the anchovy school, which varies with frequency, and is related to the scattering amplitude by:

$$
\operatorname{TS}(f)=20 \log _{10}\left|\mathbf{f}_{S}(k, \pi, 0)\right|, \quad[\mathrm{dB}]
$$

where $\mathbf{f}_{S}(k, \pi, 0)$ is the school scattering amplitude evaluated in the back scattering direction (i.e. in the direction counter to the incident field).

Note the correspondence between data and modeling results. Multiple scattering interactions between fish lead to the disappearance, in this case, of an observable resonance peak at the resonance frequency for an individual anchovy. Interference between the back scattered fields from the different fish give rise 


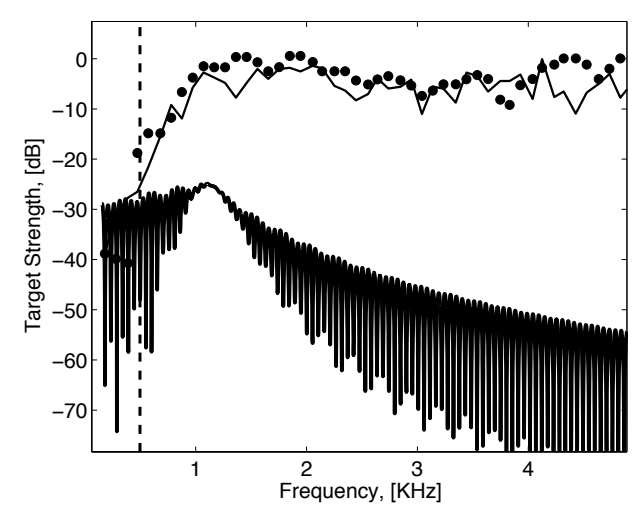

(a)

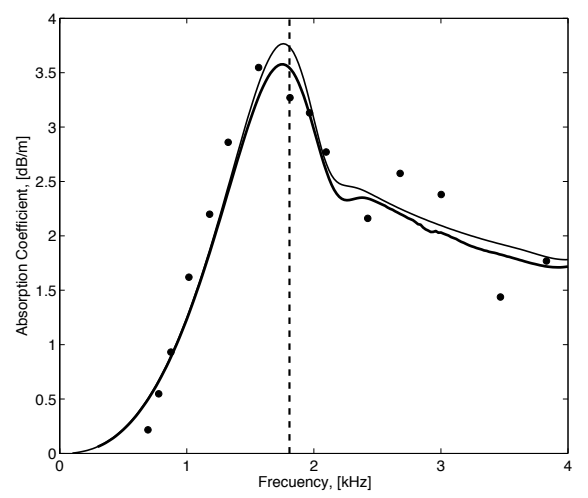

(b)

Figure 1: Back and forward scattering from fish schools. (a) Target strength of a school of anchovy. Data points “ø” from Holliday (Ref. 4). Solid line - school scattering model. (b) Absorption coefficient of school of sardines. Data points “॰” are from Diachok (Ref. 5). Solid line - school scattering model. Vertical dashed lines - individual fish resonance frequency. Thick solid line effective medium approach.

to frequency varying (Bragg scattering) peaks and troughs. Their exact location varies from shot to shot, but the overall variations are replicated by the scattering model.

The thick curve in Figure 1(a), which has many oscillations, results from using the same fish and school parameters, but treating the school as an effective medium whose sound speed variation is given by:

$$
k_{e}^{2}=k^{2}+4 \pi n \mathbf{f},
$$

where $\mathbf{f}=a /\left[\left(\omega_{0}^{2} / \omega^{2}\right)-1+i \delta_{B}\right]$ is the "scattering amplitude" of the swim bladder of an individual fish. At very low frequencies the effective medium approach can accurately represent the back scattering but typically fails, in back scattering, at frequencies at and above which the acoustic wavelength is shorter than the average distance between adjacent scatterers. This occurs in this case where, using the parameters which Holliday specifies, the effective medium completely fails to give an accurate representation.

Figure 1(b) shows a comparison with forward scattering data, obtained by Diachok (Ref. 5), from schools of sardines. The input parameters to the scattering model were taken from Diachok's article. The vertical axis shows the absorption coefficient of schools of sardines, which varies with frequency, and is related to the scattering amplitude by the extinction cross-section $\sigma_{e}$, which is defined by way of the "extinction" theorem, i.e.,

$$
\sigma_{e}=\frac{4 \pi}{k} \operatorname{Im}\left\{\mathbf{f}_{s}(k, 0,0)\right\},
$$

where "Im" denotes the "imaginary part of," and $\mathbf{f}_{s}(k, 0,0)$ is the school scattering function evaluated in the direction of the incident field.

Note that the Bragg scattering peaks and troughs typically seen in back scattering case [i.e., figure 1(a)] are not seen in this forward scattering case. The predictions of the school scattering model again clearly replicate the data. This type of forward scattering analysis can be used to estimate fish abundance in schools, by measuring the downward shift in the main resonance frequency, i.e., $f_{0}^{\prime} / f_{0}$. 
In figure 1(b), the thick line is the prediction of the effective medium approach. There is seen to be close correspondence between both the school scattering model and the effective medium results. The limitation of the effective medium results seen in back scattering, at frequencies at and above which the acoustic wavelength is shorter than the average distance between adjacent scatterers, does not apply in forward scattering. Table 1 shows a comparison of both the school model and the effective medium model with data from Diachok (Ref. 5).

\begin{tabular}{|c|c|c|c|c|c|}
\hline Measured $f_{0}$ & Depth & $f_{0} / f_{0}$ & $r$ & Diachok & School model / Effective medium \\
\hline $1.7 \pm 0.1 \mathrm{kHz}$ & $65 \mathrm{~m}$ & 0.6 & $0.8 L$ & $N=5,000$ & $N=7,000$ \\
\hline
\end{tabular}

Table 1: A direct comparison between the data interpretations described by Diachok (Ref. 5) and the present analysis. $r$ is the average nearest-neighbor spacing between fish, and $L$ is the fish length.
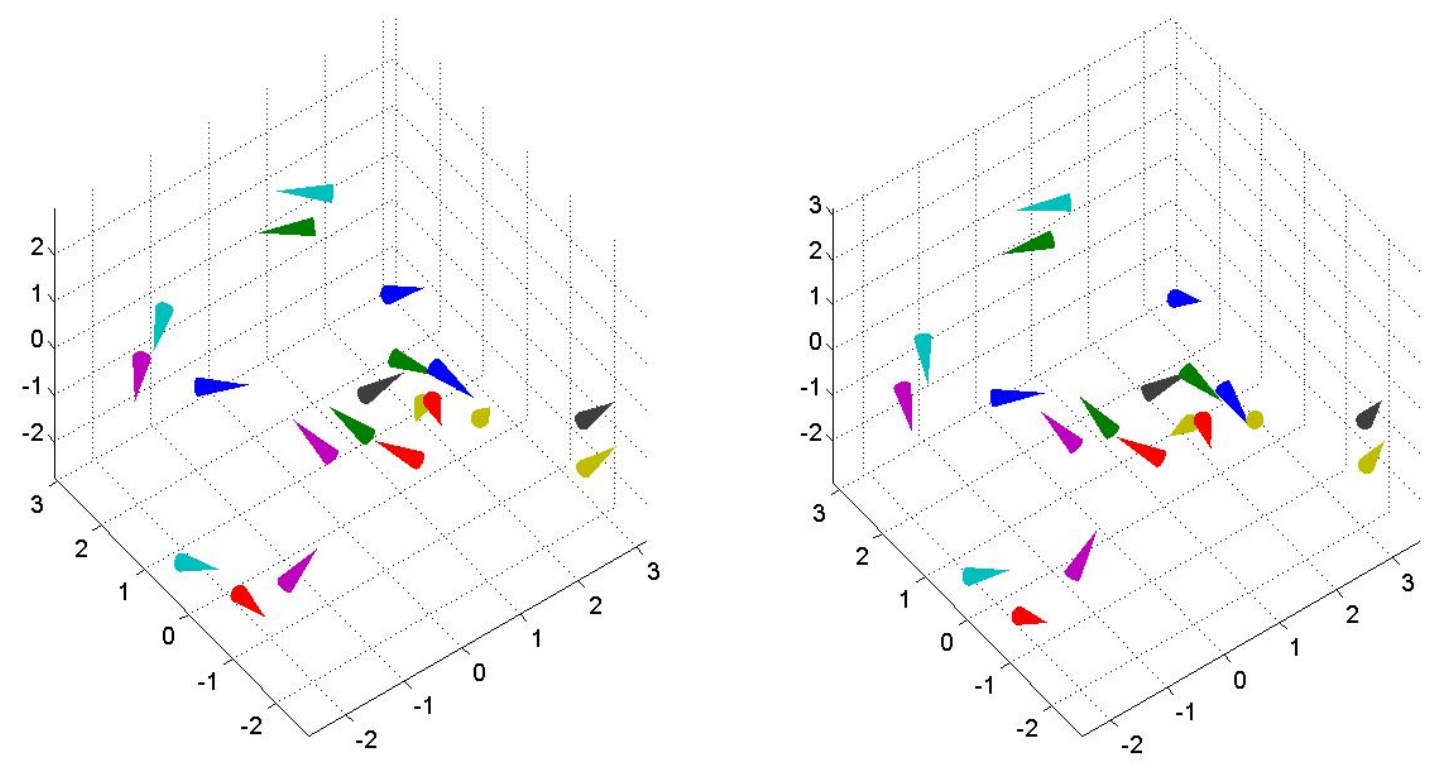

Figure 2: Two consecutive snapshots of fish positions from a movie representing the behavior of 20 fish in a school, using biological modeling based upon the article by Couzin et al, (Ref. 6). Each cone represents a fish, and the point of the cone represents the fish direction.

(b) Statistical variations of scattering from a simulated fish school.

Figure 2 shows a representation of a behavioral model for fish school behavior that has been developed, based upon the article by Couzin et al, (Ref. 6). It presents a self-organizing model of group formation in three-dimensional space, which can be used to predict the spatial dynamics fish schools. It incorporates different rules for behavior by individual fish and the school as a whole, based upon interactions between individual neighboring fish, collective memory, and other factors. The model indicates four main modes of motion for fish schools: "loosely spaced and randomly directed," "parallel" direction, "strictly parallel" direction, and "toroidal" motion.

Figure 3 is a simulation which shows the variation of target strength of a school of 250 fish swimming in "parallel" mode. This curve was produced using the steady-state school scattering model (Ref. 1). The resonance frequency of an individual fish within the school is $392 \mathrm{~Hz}$, and the peak target strength 


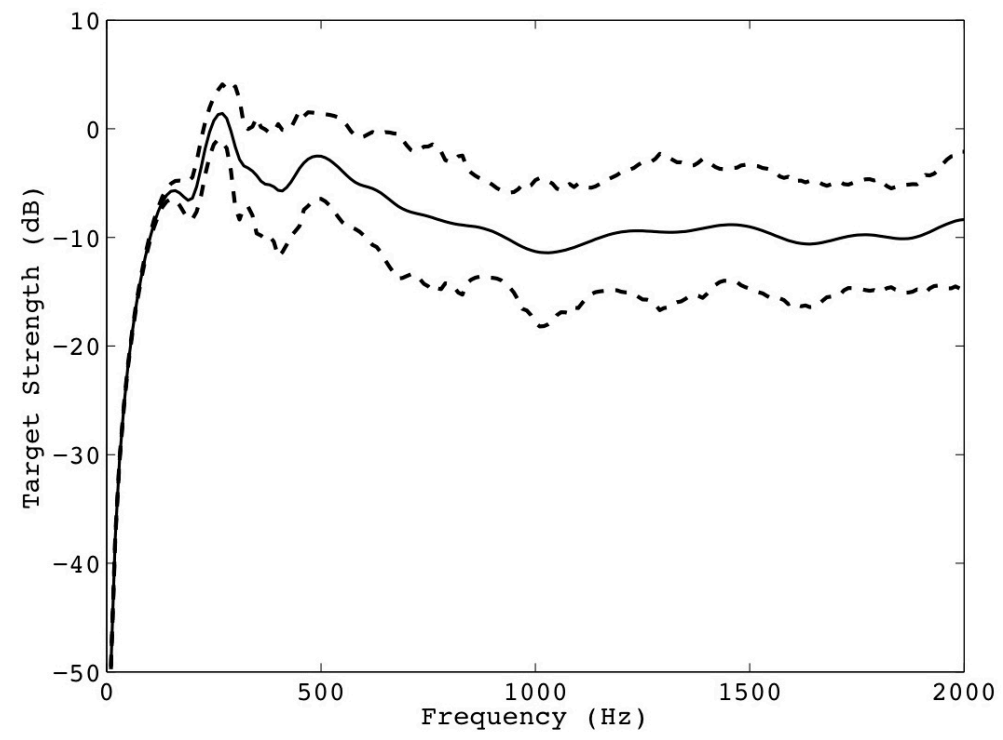

Figure 3: Variation of target strength of school of 250 fish, of length $L=40 \mathrm{~cm}$, at $50 \mathrm{~m}$ depth. Using the biological model of fish school behavior based upon Ref. 6, a "movie" of $50 \mathrm{~s}$ was produced, consisting of 500 frames, spaced 0.1 s apart, of fish swimming in a "parallel" configuration. The solid curve shows the average target strength, the upper and lower dashed lines represent the standard deviation of the target strength based upon the 500 frames.

of the school obtained by incoherently adding the target strength values of the individual fish (not shown), is $\approx 8 \mathrm{~dB}$. From figure 3 it may be seen that the main peak is shifted downwards from the 392 $\mathrm{Hz}$ value, by the multiple scattering interactions between the fish, to a value $\approx 250 \mathrm{~Hz}$, and has a reduced peak target strength of $\approx 2 \mathrm{~dB}$. This type of frequency downshift, and peak reduction, behavior is consistent with previous theoretical and experimental studies (Refs. 1, 3, 5). It may also be seen, at and near this resonance region, that the solid line has had imposed upon it several peaks and troughs, which may be due to Bragg-type interference interactions between the scattered fields from the different fish. Below this region, at frequencies up to about $125 \mathrm{~Hz}$, the motion of the fish within the school causes essentially no variations in the target strength, as may be seen by the two dashed lines being almost exactly co-incident with the solid line. In the resonance region itself the standard deviation (dashed) lines lie at about $\approx \pm 3-4 \mathrm{~dB}$ above and below the mean curve. As the frequency increases, this range also increases until, at about $750 \mathrm{~Hz}$, it reaches a fairly consistent value of $\approx \pm 5-6 \mathrm{~dB}$ for the remainder of the frequency range displayed.

\section{REFERENCES}

(1) C. Feuillade, R.W. Nero and R. H. Love, “ A low frequency acoustic scattering model for small schools of fish,” J. Acoust. Soc. Am., 99, 196-208 (1996).

(2) R. H. Love, "Resonant acoustic scattering by swim bladder-bearing fish," J. Acoust. Soc. Am. 64, 571-580 (1978).

(3) M. Raveau and C. Feuillade, "Sound extinction by fish schools: Forward scattering theory and data analysis," J. Acoust. Soc. Am. (submitted, and in review).

(4) D. V. Holliday, "Resonance structure in echoes from schooled pelagic fish," J. Acoust. Soc. Am., 
51, 1322-1332 (1972).

(5) O. Diachok, "Effects of absorptivity due to fish on transmission loss in shallow water," J. Acoust. Soc. Am., 105(4), 2107-2128 (1999).

(6) I. D. Couzin, J. Krause, R. James, G. D. Ruxton and N. R. Franks, ”Collective memory and spatial sorting in animal groups.’J. theor. Biol. 218(1), 1-11 (2002).

\section{IMPACT/APPLICATIONS}

Previous work has shown that estimation of transmission loss due to extinction by fish schools in the water column should be based upon forward scattering modeling of scattering from these schools, while the use of back scattering results can introduce significant errors. Work completed during the present reporting period has shown, in addition, that much care should be taken when using an effective medium approach to represent scattering from these objects. In back scattering, depending on the frequency, fish spacing, and school depth, an effective medium treatment may fail very significantly. However, in forward scattering, it is shown that effective medium methods are stable, and can give substantially the same results, at much less computational cost, as a full multiple scattering treatment.

The application of a collective model of fish behavior, based upon observed biological behavior, has enabled a method of predicting statistical fluctuations in target strength from fish schools. This may be useful in estimating clutter statistics for these objects in relation to SONAR applications.

\section{TRANSITIONS}

None at the present time.

\section{RELATED PROJECTS}

Experimental and other work by other participants in the ONR BRC, Fish Acoustics program.

\section{PUBLICATIONS}

(1) M. Raveau and C. Feuillade, " Sound extinction by fish schools: Forward scattering theory and data analysis," J. Acoust. Soc. Am. [submitted, and in review, significantly revised during review period]. (2) C. Feuillade and M. P. Raveau, "Sound absorption by fish schools: Forward scattering theory and data analysis," J. Acoust. Soc. Am. 134, 3990 (2013) [Abstract]

(3) M. P. Raveau and C. Feuillade, "Time domain investigations of acoustical scattering from schools of swim bladder fish,” J. Acoust. Soc. Am. 135, 2177 (2014). [Abstract]

(4) C. Feuillade, "Recent developments in acoustical scattering from ensembles of swim bladder fish," to appear in Proceedings of FIA2014, Valdivia, Chile, Dec. 1-3, 2014. [Proceedings article, in press]

(5) M. P. Raveau and C. Feuillade, "Low frequency acoustic response of a school of fish," to appear in Proceedings of FIA2014, Valdivia, Chile, Dec. 1-3, 2014. [Proceedings article, in press]

(6) S. E. Alfaro, J. A. Cellio , M. P. Raveau and V.Rojas, "Acoustic scattering of a simulated school from collective behavior in animals," to appear in Proceedings of FIA2014, Valdivia, Chile, Dec. 1-3, 2014. [Proceedings article, in press] 